\title{
Statistical Properties of the Spatial Distribution of Galaxies
}

\author{
N. Yu. Lovyagin 1 \\ St.-Petersburg State University, Universitetskij pr. 28, St.-Petersburg, 198504 Russia
}

Astrophysical Bulletin, 2009, Vol. 64, No. 3, pp. 217-228.

The original publication is available at www.springerlink.com:

http://www . springerlink. com/content/m04u0814r1706511.

\begin{abstract}
The methods of determining the fractal dimension and irregularity scale in simulated galaxy catalogs and the application of these methods to the data of the $2 \mathrm{dF}$ and $6 \mathrm{dF}$ catalogs are analyzed. Correlation methods are shown to be correctly applicable to fractal structures only at the scale lengths from several average distances between the galaxies, and up to (10-20)\% of the radius of the largest sphere that fits completely inside the sample domain. Earlier the correlation methods were believed to be applicable up to the entire radius of the sphere and the researchers did not take the above restriction into account while finding the scale length corresponding to the transition to a uniform distribution. When an empirical formula is applied for approximating the radial distributions in the samples confined by the limiting apparent magnitude, the deviation of the true radial distribution from the approximating formula (but not the parameters of the best approximation) correlate with fractal dimension. An analysis of the $2 \mathrm{dF}$ catalog yields a fractal dimension of $2.20 \pm 0.25$ on scale lengths from 2 to $20 \mathrm{Mpc}$, whereas no conclusive estimates can be derived by applying the conditional density method for larger scales due to the inherent biases of the method. An analysis of the radial distributions of galaxies in the $2 \mathrm{dF}$ and $6 \mathrm{dF}$ catalogs revealed significant irregularities on scale lengths of up to $70 \mathrm{Mpc}$. The magnitudes and sizes of these irregularities are consistent with the fractal dimension estimate of $D=2.1-2.4$
\end{abstract}

\section{INTRODUCTION}

The spatial distribution of galaxies bears signatures of both the initial conditions in the early Universe and the evolution of the primordial density perturbations. An analysis of various galaxy samples performed using the two-point correlation function showed that this function has a power-law form $\xi(r)=\left(r_{0} / r\right)^{\gamma}$ on scale lengths ranging from 0.01 to $10 \mathrm{Mpc}$ (hereafter we adopt a Hubble constant of $H_{0}=100 \mathrm{~km} / \mathrm{s} / \mathrm{Mpc}$ ) with a slope of $\gamma=1.77$ and the parameter $r_{0}=5 \mathrm{Mpc}$ [1]. It has long been considered that the scale of the $r_{0}$ parameter is the typical irregularity scale length, and the distribution of galaxies becomes uniform starting from the scale length of $r_{0}=5 \mathrm{Mpc}$. However, the discovery of structures with the scale lengths of several tens and hundreds Mpc [2] in recent surveys has cast doubt upon this hypothesis.

In this context, the problems of applicability limits and reliability of the correlation methods of the analysis of spatial distribution of galaxies, and finding new methods for describing large and very large structures acquire special importance.

At present, two kinds of data on the galaxy redshifts are of great importance.

\footnotetext{
${ }^{1}$ E-mail: lovyagin@mail.com
} 
- The first kind are the redshift catalogs covering large areas (solid angles) of the sky, but limited to small redshifts (up to $z \lesssim 0.5)$ ( $2 \mathrm{dF}, 6 \mathrm{dF}$, SDSS, etc.). Such catalogs can be analyzed via applying the correlation methods to determine the fractal dimension.

- The second kind is represented by the deepfield catalogs of photometric redshifts. Such studies cover small solid angles (of the order of $1^{\circ} \times 1^{\circ}$ ), but extend to much larger redshifts $z>1$ (up to 6) (COSMOS, HDF, HUDF, FDF and others). Correlation methods are difficult to apply to such catalogs due to the small radius of the largest sphere that fits entirely inside the small solid angle considered.

However, both kinds of catalogs can be used to analyze the radial distribution of galaxies, built upon a sample confined by the limiting apparent magnitude. This method not only removes the restriction on the size of the largest sphere thereby significantly increasing the attainable research scale lengths, but it can also be applied to all galaxies in the catalog and not only to those in a volume-limited sample thereby increasing the number of objects studied. An analysis of fluctuations in the radial distribution of galaxies can be used to determine both the sizes and the amplitudes of the largest structures in the galaxy sample considered.

In this paper we analyze two methods of statistical analysis of structures - a determination of the fractal dimension, and an analysis of radial distributions. Despite the fact that our analysis is limited to the $2 \mathrm{dF}$ and $6 \mathrm{dF}$ catalogs, we constructed our simulated lists with two kinds of catalogs (covering large and small solid angles on the sky).

In this paper we make use of our own software, developed to simulate three-dimensional catalogs of galaxies and to perform statistical analysis of both real and simulated samples. It is a $\mathrm{C}++$ library of functions (so far, without a user interface). We are currently preparing its description, which will be made available, along with the source code, at our web site. The software covers a somewhat broader scope of problems than that described in this paper, and will be a basis for a future package meant for comprehensive statistical analysis of the spatial distribution of galaxies.

\section{METHODS USED TO ANALYZE THE STRUCTURES}

\subsection{Estimating the Fractal Dimension}

Fractal dimension is estimated using the method of conditional density in spheres (the total correlation function in spheres). The definitions of the total and reduced correlation functions and a detailed description of their properties can be found in [2]. We chose the method of conditional density in spheres for the reasons stated by Vasil'ev [3]. He showed that this method is, on the one hand, sufficiently fast (compared to the method of cylinders), and, on the other hand, sufficiently accurate (the conditional density in spheres is, unlike the conditional density in shells, less subject to fluctuations) and, moreover, it can be applied to fractal structures (unlike the method of reduced two-point correlation function, which is built assuming uniform distribution inside the sample).

The idea of the method consists of constructing a dependence of the number of points $N(r)$ inside a sphere of radius $r$, averaged over spheres centered on all the points of the set. Only a portion of the set is considered, therefore the averaging should be performed only over the spheres that fit completely inside the set. The dimension is computed by the conditional number 
density $2 n(r)=N(r) /\left(4 / 3 \pi r^{3}\right)$ in logarithmic coordinates, where the slope of the line must be equal to the fractal dimension $D$ minus three, because the expected behavior is $n(r) \propto r^{D-3}$.

\subsection{Analysis of Radial Distributions}

Radial distribution is such a dependence $N(z)$, that

$$
\mathrm{d} N(z, \mathrm{~d} z)=N(z) \mathrm{d} z,
$$

where $\mathrm{d} N$ is the number of galaxies with redshifts between $z$ and $z+\mathrm{d} z$. The construction of such a distribution involves counting the number of galaxies $\Delta N(z, \Delta z)$ inside a spherical shell of thickness $\Delta z$, with midradius lying at the distance corresponding to redshift $z$, i.e., formula (11) transforms into

$$
\Delta N(z, \Delta z)=N(z) \Delta z .
$$

Thus, the $N(z)$ distribution can be built in bins with a certain chosen step in $\Delta z$. Traditionally, the $\Delta N(z, \Delta z)$ variable - the number of galaxies in shells - is plotted on the curves of radial distribution.

For magnitude-limited catalogs the radial distribution $N(z)$ is approximated by the following empirical formula (see, e.g., [4, 5]):

$$
N(z)=A z^{\gamma} \exp \left(-\left(\frac{z}{z_{c}}\right)^{\alpha}\right)
$$

Here the three parameters $\gamma, z_{c}$ and $\alpha$ are independent from each other and $A$ is the normalizing factor (the integral of radial distribution is normalized to the total number of galaxies in the sample):

$$
\int_{0}^{\infty} N(z) \mathrm{d} z=\int_{0}^{\infty} A z^{\gamma} \exp \left(-\left(\frac{z}{z_{c}}\right)^{\alpha}\right) \mathrm{d} z=\frac{A z_{c}^{\gamma+1} \Gamma\left(\frac{\gamma+1}{\alpha}\right)}{\alpha}=N,
$$

where $N$ is the total number of galaxies and $\Gamma(x)$ is the (complete) Euler Gamma-function. However, it is impossible, when searching for the best approximation of the radial distribution, to compute the $A$ (3) ; due to the fluctuations we have to search for it in the interval from $A-\sqrt{A}$ to $A+\sqrt{A}$.

The approximation is performed via the least squares method, i.e., one must search for the parameter values that minimize the sum of squared residuals. The classical least squares method cannot be applied as the approximating function is not linear in parameters. However, a "straightforward" minimization using the fastest (gradient) descent method is also extremely inefficient, as the minimum is indistinct and it may take a computer several days to several months to find it. That is why we employ the grid search method, where the grid mesh and search domain are reduced at each successive iteration.

After finding the best-fit parameters, the domains of irregularities are identified on the curve of relative fluctuations:

$$
\sigma_{N}=\frac{N_{o b s}-N_{\text {theor }}}{N_{\text {theor }}},
$$

\footnotetext{
${ }^{2}$ Terms "density" and "concentration" are synonyms in this sense, since the concentration is the density of point sources with the unit mass.
} 
where

$$
\begin{aligned}
N_{\text {obs }} & =N\left(z_{i}, \Delta z\right) \\
N_{\text {theor }} & =\left.A z^{\gamma} \exp \left(-\left(\frac{z}{z_{c}}\right)^{\alpha}\right)\right|_{z=z_{i}} .
\end{aligned}
$$

We can thus interpret any fluctuation exceeding the Poisson noise level of $\sigma_{N}>3 \sigma_{p}$, as a structure, where ${ }^{3} \sigma_{p}=1 / \sqrt{N_{\text {theor }}}$, because in a fractal distribution the characteristic fluctuation is increased by $\sigma_{\xi}$, which can be computed based on the value of the two-point correlation function $\xi(r)$ :

$$
\sigma_{\xi}^{2}=\frac{1}{V^{2}} \int_{V} \mathrm{~d} f V_{1} \int_{V} \mathrm{~d} f V_{2} \xi\left(\left|r_{1}-r_{2}\right|\right),
$$

where $V$ is the volume of the set $[6,7,8]$.

\section{CATALOGS USED}

\subsection{The 2dF Catalog}

The $2 \mathrm{dF}$ catalog $2 \mathrm{dF}$ [9], or, more precisely, its 2dFGRS subsample, which includes the data on the redshifts of galaxies, contains a total of 245591 objects, of which about 220 thousand have sufficiently accurately measured redshifts. The magnitude limits in the $J$-band, corrected for the Galactic extinction, are $14.0<m_{J}<19.45$. Most of the galaxies have redshifts $z<0.3$. The catalog is available at http://magnum.anu.edu.au/ TDFgg.

The galaxies of the catalog concentrate in the sky in two continuous strips extending along the right ascension, and in randomly scattered small areas. About 140 thousand galaxies are located in the Southern strip, and about 70 thousand galaxies, in the Northern strip.

\subsection{The $6 \mathrm{dF}$ Catalog}

The 6dFGS catalog is an all-sky spectroscopic survey at Galactic latitudes $|b|>10^{\circ}[10$, 11, 12. Observations began in 2003 and were made using a multichannel spectrograph (they have not yet been completed at the time of writing this paper). The catalog is available at http://www-wfau.roe.ac.uk/6dFGS. In this paper we use the second data release of the catalog, which contains 83014 galaxies with known equatorial coordinates. Of these, 71627 objects have sufficiently reliably determined redshifts. The survey has been completed in three sky areas. In this paper we use a sample of galaxies with known $R$-band magnitudes.

\section{SIMULATED GALAXY CATALOGS}

To test the reliability and accuracy, and to identify the applicability limits of the methods, they must be applied to simulated catalogs. To this end, we generate catalogs that simulate not only the spatial distribution of galaxies (uniform and fractal), but also the distribution of their absolute magnitudes (i.e., the luminosity function of galaxies). Such catalogs can be subjected to both the correlation analysis (determination of the fractal dimension) in a volume-limited

\footnotetext{
${ }^{3}$ Here we use $N_{\text {theor }}$ and not $N_{o b s}$, because the latter may be equal to zero.
} 
sample in a large solid angle, and to the analysis of the radial distribution in a magnitudelimited sample either in a large or in a small solid angle.

Moreover, we use the MersenneTwister pseudorandom number generator to generate random quantities (space positions and absolute magnitudes of galaxies). This generator, unlike the standard linear congruent generator, produces far less correlated numbers and it is considered suitable for the use of Monte-Carlo method [13.

In this paper we analyze a fractal model of the real distribution of galaxies parametrized by the fractal dimension and the parameters of the luminosity function. This model describes the power-law nature of the observed correlations of the distribution of galaxies in real catalogs.

\subsection{Spatial Distribution of Galaxies}

We use three models of the spatial distribution of galaxies.

Uniform distribution. The coordinates of each point of the set are generated as three random numbers uniformly distributed in the $[0,1]$ interval (and hence the entire set is contained in the $[0,1] \times[0,1] \times[0,1]$ cube) .

Cantor dust (more precisely, its generalization to the three-dimensional case). The zero generation of this set coincides with the $[0,1] \times[0,1] \times[0,1]$ cube. Each edge of the cube is then subdivided into $m$ equal parts, i.e., the entire cube is subdivided into $m^{3}$ identical subcubes, and for each such subcube the probability $p$ of its "survival" in the next generation is defined. The next generation consists of the set of "surviving" subcubes, and the algorithm is then reiterated for each such subcube. The final set is the limit obtained as the number of the generation becomes infinite: in each generation the edge of the cube becomes shorter by a factor of $m$ and tends to 0 as $\frac{1}{m^{n}}$, , i.e., the subcubes contract to points. In case of a real distribution the process should be terminated at a certain generation $n$. A point is chosen inside each of the subcubes "surviving" in the last generation. The coordinates of this point are random numbers uniformly distributed along the projections of the edges of the subcube onto the coordinate axes.

The theoretical dimension of such a set is known to be given by the formula

$$
D=\log _{m}\left(p m^{3}\right) \text {. }
$$

In our case we use the given dimension $D$ to compute the probability $p=m^{D-3}$.

Gaussian random walk and its generalization with the possibility of generating sets of $2 \leqslant$ $D \leqslant 3$. dimension. The first point coincides with the coordinate origin $(0,0,0)$. In the classical case each successive point is obtained from the previous point by adding to its every coordinate a normally distributed random number with zero mean and unit variance.

The generalization that we propose here for the first time consists of the following: at each stage we generate two points instead of one with a certain probability $w$. A more accurate description of the algorithm uses the term "generation". The zero generation coincides with the coordinate origin $(0,0,0)$. Every next generation is obtained from the previous generation in accordance with the following rule: for each point of the previous generation one or two points of the new generation are generated, like in the classical case, by adding normally distributed random numbers to the coordinates of the previous 


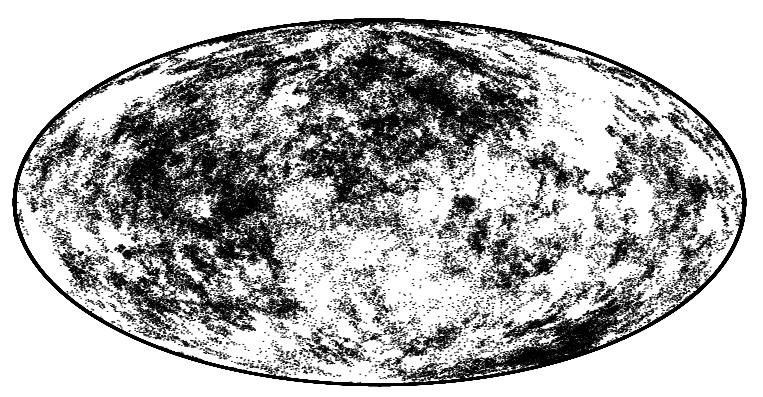

Fig. 1. The Hummer-Aitoff projection of the generalized Gaussian random walk with $w=10^{-4}$. Our sample is a sphere with a smaller radius (see Section 5.2).

point so that the probability of generating one or two points is equal to $1-w$ and $w$, respectively. The algorithm is reiterated for each point of the new generation. The case of $w=0$ corresponds to classical Gaussian random walk. Figure 1 shows the corresponding set with $w=10^{-4}$.

\subsection{The Absolute Magnitude Distribution of Galaxies}

We generate the absolute magnitude distribution of galaxies in simulated catalogs in a way to make it consistent with the galaxy luminosity function - the Schechter function. We adopt the form of this function from [14, 15]:

$$
S(M)=0.92 \phi_{0} \exp \left(-0.92(\alpha+1)\left(M-M^{*}\right)-\exp \left(-0.92\left(M-M^{*}\right)\right)\right) .
$$

The probability density $f_{M}$ of the absolute magnitude as a random quantity is given by the normalized variant of the Schechter function:

$$
f_{M} \mathrm{~d} M=\frac{S(M) \mathrm{d} M}{\int_{M_{\min }}^{M_{\max }} S(M) \mathrm{d} M} .
$$

We adopt the parameters of the luminosity function from the studies of the $2 \mathrm{dF}$ catalog [16] $\left(M^{*}=-19.67, \phi_{0}=0.0164, \alpha=-1.21\right)$, since we develop our simulated catalogs as the models of the $2 \mathrm{dF}$ catalog.

\section{RESULTS OF THE ANALYSIS OF SIMULATED FRACTAL STRUCTURES}

\subsection{Simulated Galaxy Catalogs}

We generated uniformly distributed points for the Cantor sets with the dimensions of 2.0 and 2.6, and for the Gaussian random walk with $w=10^{-4}$ (the fractal dimension of this set is estimated at $2.4 \pm 0.3$ ). The number of generated points exceeded $7.5 \cdot 10^{7}$ for all cases. This 
procedure is followed by the determination of the center of a sphere containing the same number of points $2 \cdot 10^{7}$ for each variant (these restrictions are determined by the available computer resources). The resulting sphere serves as a model of the observed part of the Universe with the observer located at its center.

Finding the center is a necessary procedure, because we have to make sure that the set has been generated completely in the selected region, i.e., our volume contains no voids due to the finite size of the generated set, rather than due to its fractal structure. Such voids may bias the results obtained by analyzing the model. To ensure this, the center is found as the locus of the highest concentration, or, more precisely, the adopted center coincides with such a point of the set, where the radius of the sphere centered on it and containing the required number of points would be minimal among all spheres for all generated points. However, the procedure of finding such a point is too time-consuming, making it impossible to use the exhaustive search algorithm. Instead, the set is subdivided into cubes, and instead of counting the number of points in the sphere, we count the number of cubes in the sphere with the weights equal to the number of points in the cube.

Each galaxy inside the selected sphere is assigned with an absolute magnitude distributed in accordance with the Schechter law (5). By setting the parameter $\phi_{0}$ (see Section 4.2) one can establish a unique relation between the number of galaxy points $N_{0}$ in the simulated spherically symmetric set and the radius $R_{0}$ of its bounding sphere [15]:

$$
R_{0}=\sqrt[3]{\frac{\frac{4}{3} \pi \phi_{0} \Gamma(1+\alpha, \beta)}{N_{0}}}
$$

where $\Gamma$ is the incomplete Gamma-function:

$$
\Gamma(a, z)=\int_{z}^{\infty} e^{-t} t^{a-1} \mathrm{~d} t .
$$

We can thus compare the real and simulated catalogs.

\subsection{Subsamples of Simulated Catalogs}

We generated five subsamples for each spherically symmetric simulated catalog:

- a sample bounded by the concentric sphere of smaller radius containing exactly $10^{5}$ points (this is the number that allows the fractal dimension to be computed in reasonable time by constructing a grid of models);

- a small solid-angle sample of about $10^{5}$ points, which is also used to compute the fractal dimension $(\Omega \sim 0.05 \pi)$;

- a magnitude-limited sample with $m_{l i m}=17^{m} .0$ used to construct radial distributions. For this subsample we constructed three volume-limited samples containing objects up to $z=0.013$ and $M \sim-16^{m} .3$, up to $z=0.1$ and $M \sim-20^{m} .3$, up to $z=0.13$ and $M \sim-20^{m} .9$, in order to compute the fractal dimension;

- a magnitude-limited sample covering a large solid angle $(\Omega \sim 0.3 \pi)$ to be used to construct radial distributions. For this subsample we constructed three volume-limited samples 
containing objects located up to $z=0.04$ and $M \sim-18^{m} .4$, up to $z=0.06$ and $M \sim$ $-19^{m} .3$, up to $z=0.08$ and $M \sim-19^{m} .9$, in order to compute the fractal dimension. The sample can be viewed as a model of the $2 \mathrm{dF}, 6 \mathrm{dF}$ and other similar catalogs;

- a magnitude-limited sample inside a small solid angle, to be used only for constructing radial distributions. The sample can be viewed as a model of the COSMOS, HDF and other similar catalogs.

\subsection{Conclusions Based on the Analysis of Simulated Catalogs}

\subsubsection{Conclusions concerning the efficiency of the use of the method of conditional density in spheres for determining the fractal dimension}

The dimension $D$ is determined by analyzing the conditional density function $n(r)$ in logarithmic coordinates, where it must transform from a power-law form into a linear function

$$
\lg n=A+(D-3) \lg r .
$$

However, practice shows that in reality it is not linear over the entire interval from $r_{0}$ (the minimum distance between the points) to $r_{m}$ (the radius of the greatest sphere that fits entirely inside the set). For each of such sets (a catalog), three characteristic portions can be identified on the curve of conditional density (from left to right):

- in the first portion of the curve (between $r_{0}$ and $r_{1}$ ), the decrease of $n(r)$ corresponds to dimension 0 : the corresponding radii are comparable to the minimum distance between the points of the set;

- in the second portion of the curve (between $r_{1}$ and $r_{2}$ ), $n(r)$ "Gets into operational mode", where the slope (as we expect) corresponds to the dimension;

- in the third portion of the curve (between $r_{2}$ and $r_{m}$ ) the conditional probability function behaves unpredictably, as the averaging is made over too few spheres, making this portion unsuitable for computing the dimension.

Our task is thus to identify the second portion, i.e., to find such $r_{1}$ and $r_{2}$, between which the function would behave linearly. The parameters $A$ and $D$ for this portion can then be easily estimated via the leastsquares method. Figure 2 shows examples of the conditional density curves for different cases:

- based on an analysis of sets with known Hausdorff dimension (Cantor set) - a comparison of the known and computed dimensions leads us to conclude that the method is an efficient tool for determining the dimensions of the sets:

- with a spherically symmetric configuration;

- located inside a limited solid angle;

- in volume-limited galaxy samples having a spherical configuration;

- in volume-limited samples located inside a limited solid angle. 

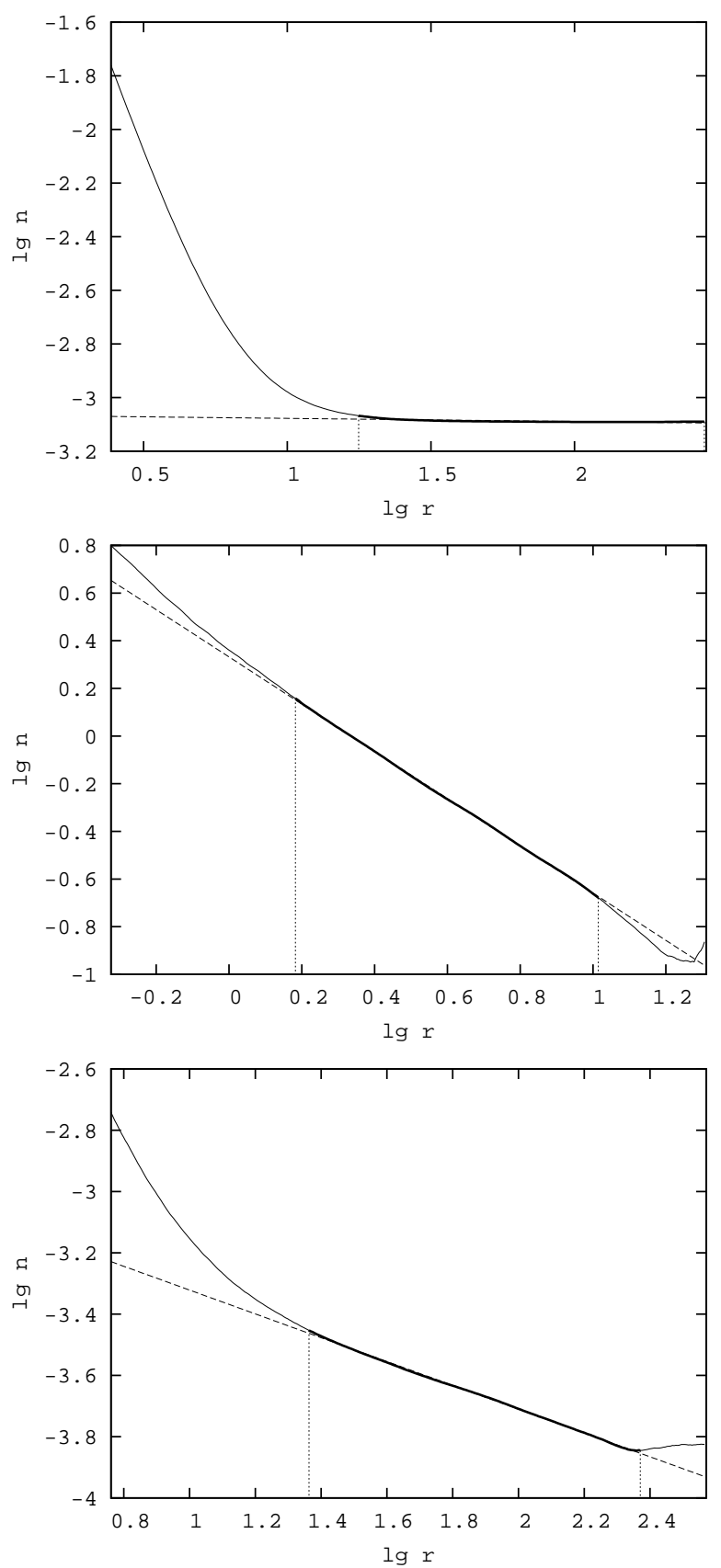

Fig. 2. Examples of conditional density curves. From top to bottom: Poisson set (the curve yields a fractal dimension of 2.99); Cantor set with a dimension of 2.6 (the computed dimension is 2.61), and Cantor set with a dimension of 2.0 (the computed dimension is 1.99). The solid lines correspond to the computed values, the dotted lines are the linear fits used to compute the dimension, and the bold line is the portion of the conditional density curve used for the linear fit. The x-values of the left- and right-hand boundaries of this portion are equal to $\log r_{1}$, and $\log r_{2}$, respectively, and the $\mathrm{x}$-value of the rightmost point of the curve is $\log r_{m}$ (these quantities are discussed in the text). 
The method can also be used to determine the dimensions of a set of galaxies in the Universe by analyzing volume-limited samples in galaxy redshift surveys in limited sky areas (a typical situation). The accuracy of the dimension determination (for sets with the same fractal dimension in all their parts) is $\pm 0.1-0.2$.

- in case of a uniform distribution, the dimension can be determined on scale lengths (the upper limit is equal to the $r_{2}$ radius) of up to $100 \%$ of the radius $r_{m}$ of the greatest sphere that fits entirely inside the set.

The radius $r_{2}$ decreases with decreasing dimension, i.e., the upper limit of scale lengths, where the fractal dimension can be determined using this method, becomes shorter and reduces at dimension 2.0 to mere $(5-20) \%$ of the radius of the greatest sphere that fits entirely inside the set. However, no clear correlation is observed due to the interference of other factors (e.g., individual features of the fractal set, lacunarity);

- for a spherical configuration the radius of the greatest sphere is equal to the survey depth radius, whereas non-spherical geometry of the sample restricts substantially the size of the greatest sphere, thereby strongly reducing the $r_{2}$ radius (down to $0.01 \%$ of the survey depth for a solid angle of $0.01 \pi)$. volume-limited samples decrease the survey depth several times;

- the conditional density curve for sets with dimensions smaller than 3.00 in the region $r>r_{2}$ can even exhibit a fictitious transition to uniformity (see, e.g., the lower panel in Fig. 2). Thus no definitive conclusions about the attainment of uniformity can be made based on the right-hand end of the conditional density curve, because even purely fractal distributions may exhibit effects of fictitious uniformity.

Hence, an analysis of catalogs like $2 \mathrm{dF}, 6 \mathrm{dF}$ and SDSS, limited in redshift by $z \lesssim 0.5$ (which corresponds to about $1300 \mathrm{Mpc}$ ) with non-spherical configurations, making it essential to select volume-limited samples, may provide conclusive results on the presence of uniformity only on the scales 30-100 times smaller (i.e., 10-40 Mpc).

Such a behavior of conditional density at $r>r_{2}$ can be explained by the fact that in this portion of the curve, the averaging of the number of points inside the sphere of radius $r$ is made over a too small number of spheres: the number of spheres centered on points of the set, and fitting entirely inside the set, decreases with increasing radius of the sphere. The extreme right point on the curve of conditional density is computed based on only one sphere (see Fig. 3). Proper statistics (suitable for fractal distribution) are accumulated only where the number of spheres amounts to $20-90 \%$ of the total number of points.

\subsubsection{Conclusions about the efficiency of the analysis of radial distributions}

We make the following conclusions about the efficiency of the analysis of radial distributions:

- concerning the parameters of the fit by the empirical formula (2) (see Fig. 4):

- this empirical formula describes the simulated distribution equally well both in the uniform case and in the fractal cases at the dimensions greater than 2.0. At smaller dimensions the fluctuations increase sharply, many "empty" bins appear, and an approximation becomes impossible; 


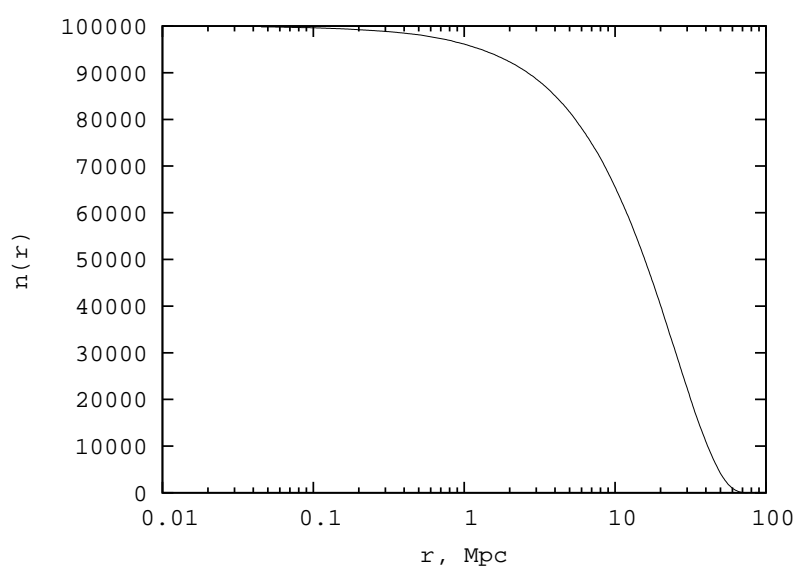

Fig. 3. The number of spheres fitting entirely inside the set as a function of the radius of the sphere (i.e., the number of spheres used for averaging the conditional density function - the number of points in these spheres - for the adopted sphere radius) for the Poisson set.

- the best-fit parameter 4 are sufficiently stable against a change in the step in $z$, except for the large (in our case, smaller than $1 / 25$ of the survey depth) steps;

- these parameters are unstable against a change in the number of points (this conclusion can be made based on large differences between the parameters inferred for the spherical configuration, and the configurations limited to different solid angles in an analysis of the Poisson set). Parameters are unstable against the choice of solid-angle samples;

- parameters show no evident correlation with the fractal dimension. It was expected that $\gamma=D-1$, but this is evidently not the case. Moreover, totally different combinations of independent parameters may result in very similar functions with approximately the same sum of squared residuals. This may indicate that the parameter set for this problem is redundant, thereby casting doubts on the unconditional adoption of the above empirical formula for approximating the radial distribution. However, in cases when a good combination of parameters (i.e., a combination that results in a small sum of squared residuals) is found, relative fluctuations of the approximations about the observed value can be analyzed. Although a small sum of squared residuals can be achieved with different parameter combinations, the corresponding curves of relative fluctuations are almost identical for all variants. Thus, the approximation by this formula makes it possible to determine the scale and magnitude of fluctuations, which allows the structures to be adequately identified using the method described in Section 2.2.

- concerning relative fluctuations:

- the higher is the amplitude of fluctuations (44), the smaller is the fractal dimension. It increases from (3-4) $\sigma_{p}$ for uniform distribution to (30-40) $\sigma_{p}$ for Cantor set with a dimension of 2.0 (see Fig. (5). The Gaussian random walk yields a somewhat smaller amplitude;

\footnotetext{
${ }^{4}$ For the true probability density of a galaxy's redshift as a random quantity, i.e., for the comparison we take not the $A$ variable from formula (3), but $A / N / \Delta z$, where $N$ is the total number of galaxies in the sample and $\Delta z$ is the adopted step.
} 

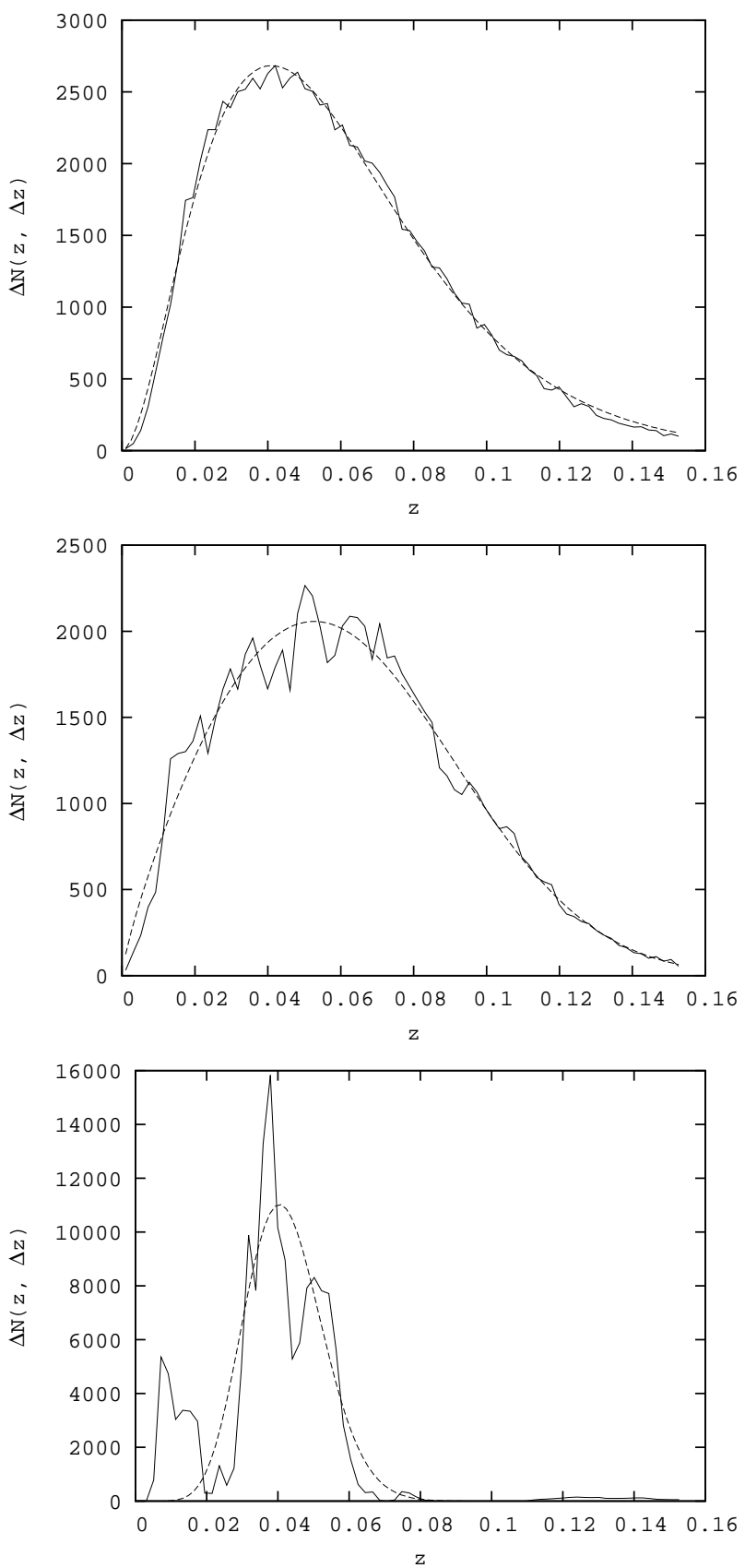

Fig. 4. Example of a radial distribution for (from top to bottom) the Poisson set, the Cantor set of dimension 2.6 and the Cantor set of dimension 2.0. $\Delta z=z_{m} / 75$ (the number of bins is 75). The solid and dotted lines correspond to the measured radial distribution and the distribution, approximated by the empirical formula, respectively. 

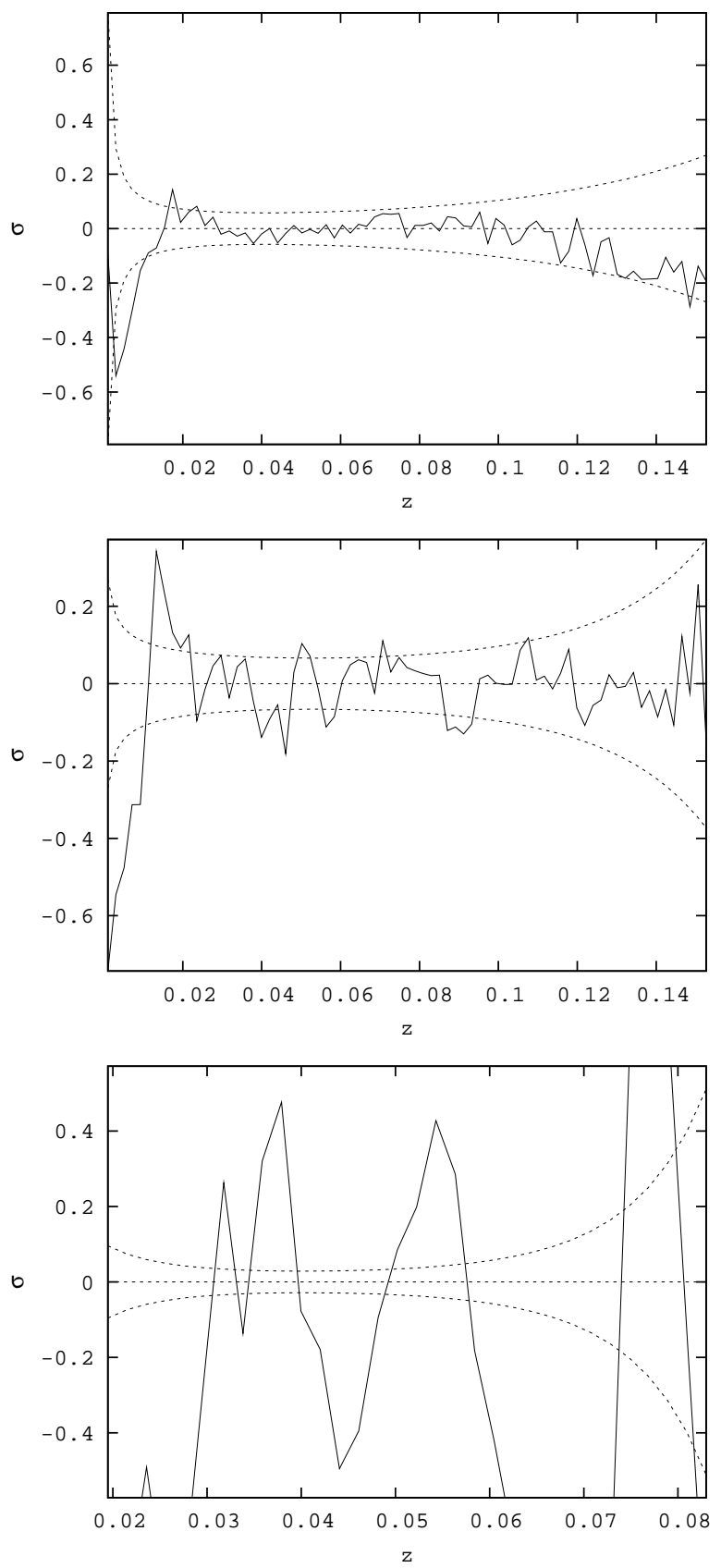

Fig. 5. Example of the curve of relative fluctuations for (from top to bottom) the Poisson set, the Cantor set with a dimension of 2.6, and the Cantor set with a dimension of 2.0. Here $\Delta z=z_{m} / 75$ (the number of bins is equal to 75). The solid lines correspond to the measured fluctuation and the dotted lines - to the $\sigma=0$ and $\pm 3 \sigma_{p}$ levels. Fluctuations exceeding this level must indicate the detection of a structure. The curve for uniform distribution exhibits two, probably fictitious, large fluctuations due to the eventual inaccuracy of the empirical formula. The same fluctuations recur on the curve for the dimension of 2.6, but they are superimposed by proper fluctuations, increasing the Poisson level, and existing due to the fractal nature of the distribution. In case of the dimension of 2.0, the magnitude of these proper fluctuations becomes very high. 
- even in case of a uniform distribution of points, the fluctuations with amplitudes above $3 \sigma_{p}$ appear at the left end of the curve of the radial distribution, and these fluctuations recur in all subsamples of the corresponding set. Apparently, the empirical formula employed does not fit sufficiently well the left end of the radial distribution (the effect of the exponential term starts too early). That is why the first two fluctuations exceeding the Poisson noise level cannot be viewed as true signatures of the structure for the sets of all dimensions;

- the number of irregularities found, their size and amplitude expressed in fractions of $\sigma_{p}$, increase with decreasing fractal dimension and decrease with decreasing solid angle;

- radial distributions can be used to determine whether irregularities are present throughout virtually the entire survey depth in the redshift interval from $10 \%$ to $60 \%$ of the survey depth $z_{m}$. The fractal dimension can be estimated by comparing the observed radial distribution with the corresponding simulated distributions.

- the step in $z$ should be neither too small nor too large, as it is in case of such an optimum step that the parameters of the approximation are more or less the same and there are few enough chance fluctuations due to "empty bins" or single points. We found the optimum number of bins to be $N=40 \div 80$, but it should be chosen individually in each particular case. Larger-than-optimum step shows up in case of the determination of the best-fit approximation of the radial distribution: the computed parameters begin to depend on the step size and the shape of fluctuations changes appreciably. Too small a step can be found at the appearance of bins containing no galaxies, and noise in the fluctuations.

\section{REDUCTION OF REAL CATALOGS}

\subsection{Computation of the Number of Dimension}

To compute the number of dimension, one has to not only select a volume-limited sample, but find an area in the sky, where the catalog has been fully completed. The $2 \mathrm{dF}$ catalog has two such areas, contains about 70000 points each. The $6 \mathrm{dF}$ catalog has three such areas, each contains at least 20000 points. Identification of a volume-limited sample leaves only about one third of all galaxies, implying that significantly less than 10000 points should remain in the areas of the $6 \mathrm{dF}$ catalog, which is evidently insufficient for computing the fractal dimension. That is why we calculate the number of dimension only for the sample of $2 \mathrm{dF}$ galaxies.

We selected two sky areas almost completely covered with observations:

\begin{tabular}{|c|c|c|c|}
\hline Interval of $\alpha$ & Interval of $\delta$ & $\Omega$ & No. of galaxies \\
\hline \hline $150^{\circ} \div 210^{\circ}$ & $-4^{\circ} \div 2^{\circ}$ & $0.034 \pi$ & 61259 \\
\hline $328^{\circ} \div 52^{\circ}$ & $-32^{\circ} \div-24^{\circ}$ & $0.057 \pi$ & 82044 \\
\hline
\end{tabular}

In each area we construct three volume-limited samples: with redshifts of up to $z=0.075$, $z=0.15$ and $z=0.2$, respectively. Figure 6 shows the cone diagrams for the volume-limited samples. 

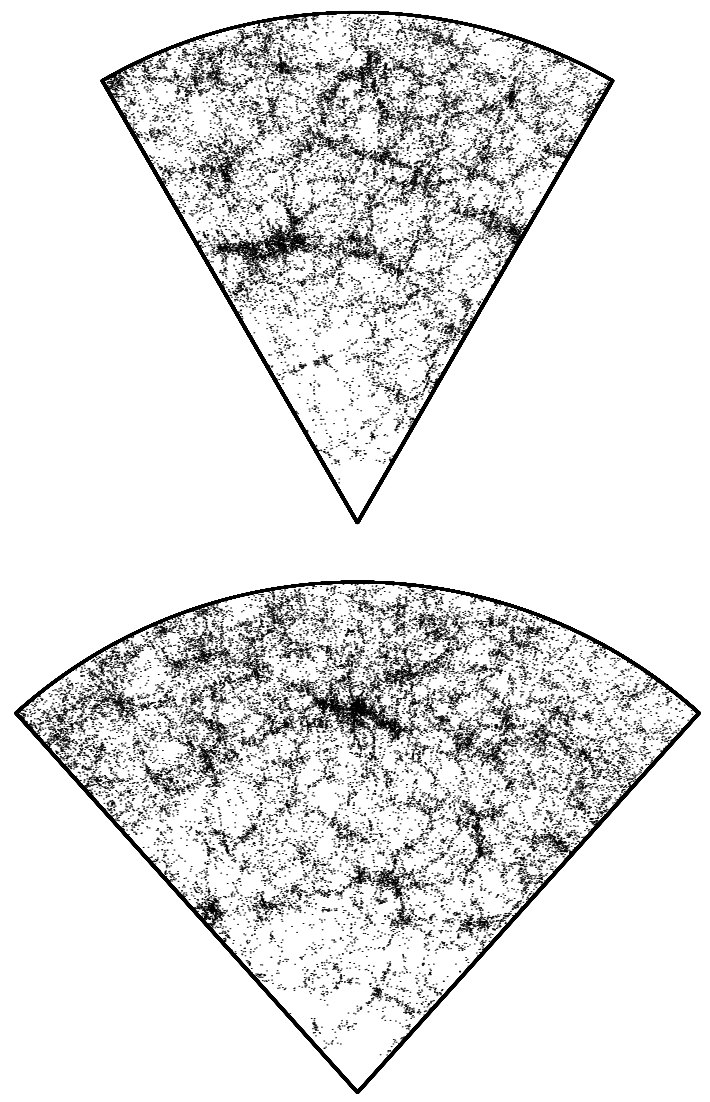

Fig. 6. Cone diagrams (in polar coordinates $(z, \alpha)$ ) of volume-limited samples up to $z_{\text {lim }}=0.15$ for the Northern (top) and Southern (bottom) domains of the $2 \mathrm{dF}$ catalog. 


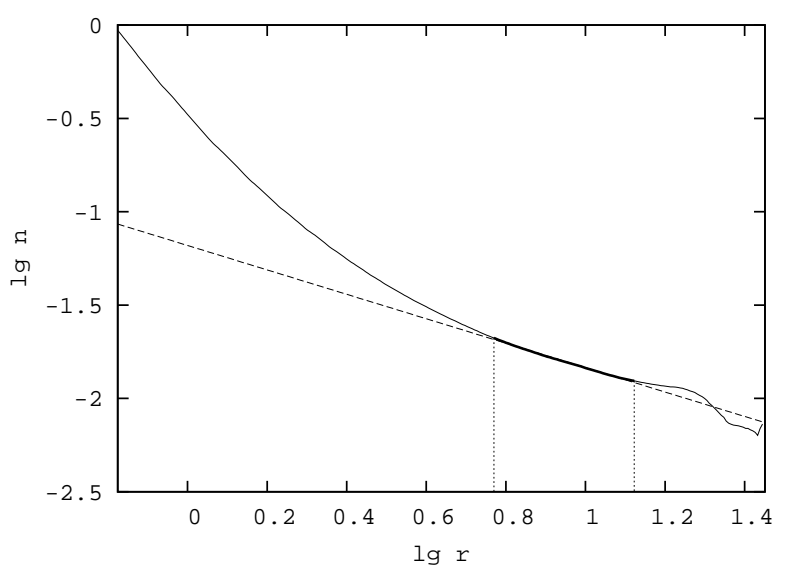

Fig. 7. Curve of conditional density for the $2 \mathrm{dF}$ catalog. volume-limited sample in the first area of up to $z=0.075$.

\subsection{Analysis of the Radial Distributions}

The analysis of radial distributions should not be necessarily restricted to completely filled sky areas. Therefore for both catalogs $(2 \mathrm{dF}$ and $6 \mathrm{dF})$ we construct three radial distributionsone for the entire set, and two for the two areas with sufficient observational coverage. The corresponding areas for the $6 \mathrm{dF}$ catalog are determined by the following parameters:

\begin{tabular}{|c|c|c|c|}
\hline Interval of $\alpha$ & Interval of $\delta$ & $\Omega$ & No. of galaxies \\
\hline \hline $290^{\circ} \div 100^{\circ}$ & $-42^{\circ} \div-23^{\circ}$ & $0.263 \pi$ & 16288 \\
\hline $150^{\circ} \div 240^{\circ}$ & $-42^{\circ} \div-23^{\circ}$ & $0.139 \pi$ & 14407 \\
\hline
\end{tabular}

For the $2 \mathrm{dF}$ catalog we select the same areas as those used to determine the dimension.

\subsection{Conclusions}

- The result of computing of the number of dimensions lead us to conclude that the fractal dimension of the set of galaxies in volume-limited samples of the $2 \mathrm{dF}$ catalog is equal to $2.20 \pm 0.25$. Irregularity scales range from $2 \mathrm{Mpc}$ to $20 \mathrm{Mpc}$ (see Fig. 7). The standard error of the dimension is greater than the error for the simulated catalogs, what suggests that the spatial distribution of galaxies is not ideally fractal, but possibly multifractal.

We also tried to find the fractal dimension for the $6 \mathrm{dF}$ catalog in areas of the best observational coverage. However, our analysis must have yielded grossly underestimated dimension (1.5 and 1.9). This effect is due to the insufficiently complete and insufficiently uniform observational coverage of the sky area considered, and different limiting redshifts $z$ in different observational areas. Both of these effects produce fictitious voids in the portion, where the dimension is determined, resulting in its underestimated value.

- As for results of the analysis of radial distributions, the main conclusion that follows from it consists in the discovery of irregularities with the amplitudes substantially exceeding not only $3 \sigma_{p}$, but even $7 \sigma_{p}$ level, in the numbers much greater than one might expect for a uniform distribution. This fact suggests a clearly non-uniform distribution of galaxies up to 300-500 and $700 \mathrm{Mpc}$. Irregularities at the right end of the radial distribution for the $6 \mathrm{dF}$ catalog only indicate that the depth in $z$ varies for different observed areas and that 

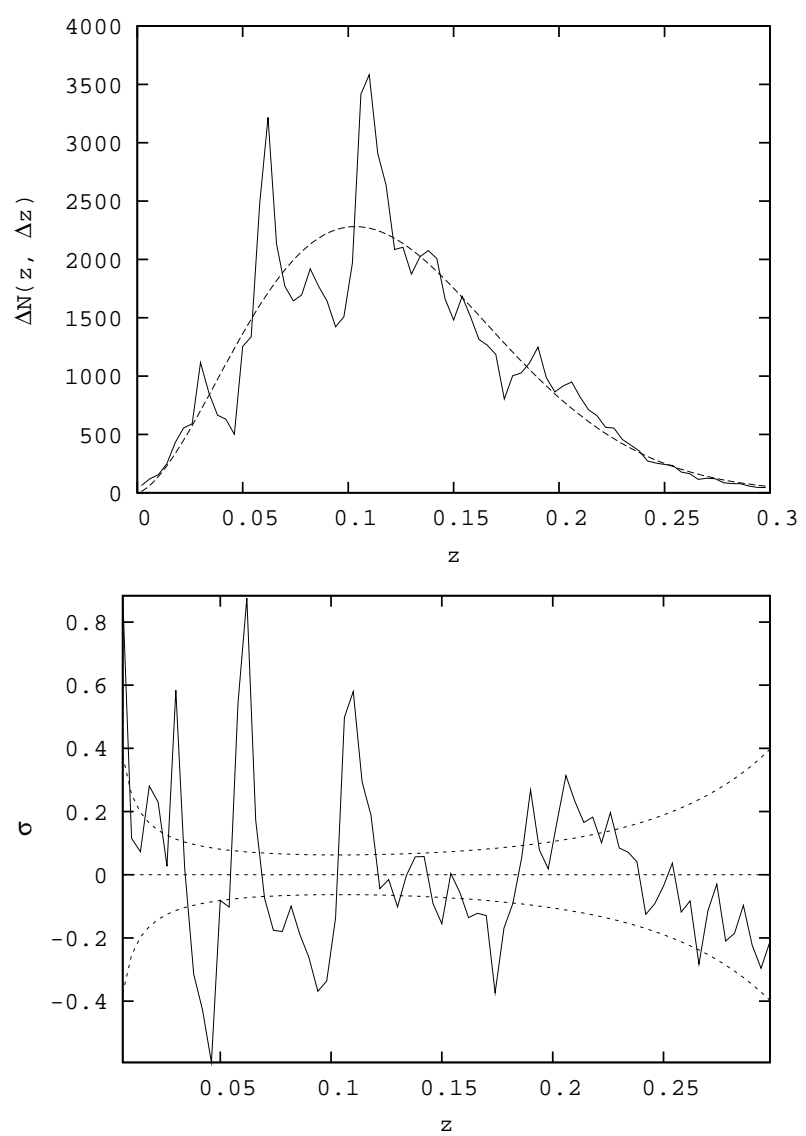

Fig. 8. Radial distribution (top) and the curve of relative fluctuations (bottom) for the $2 \mathrm{dF}$ catalog. The first domain $\Delta z=z_{m} / 75$.

the number of galaxies is insufficient at large $z$. The characteristic sizes (scale lengths) of irregularities amount to 40-70 Mpc (see Fig. 8, 9).

The irregularity amplitude is of about 6 , which corresponds to a fractal dimension greater than 2.0, but smaller than 2.6, i.e., the dimension estimate $D=2.2$, obtained using the correlation method, adequately describes the nature of irregularities in the radial distribution.

\section{CONCLUSIONS}

As a conclusion, we shall specify the following results of our analysis of simulated catalogs of the spatial distribution of galaxies, and absolute magnitude distribution of galaxies:

- correlation methods can be correctly applied only on scale lengths from several average distances between the galaxies up to (10-20)\% of the radius of the largest sphere that fits entirely inside the set. The authors of earlier studies believed that the method could be applied out to the entire radius, and the above $10 \%$ restriction, which applies to all distributions but uniform, was not taken into account when determining the scale length where the distribution becomes uniform (see, e.g., [17, [18]) 

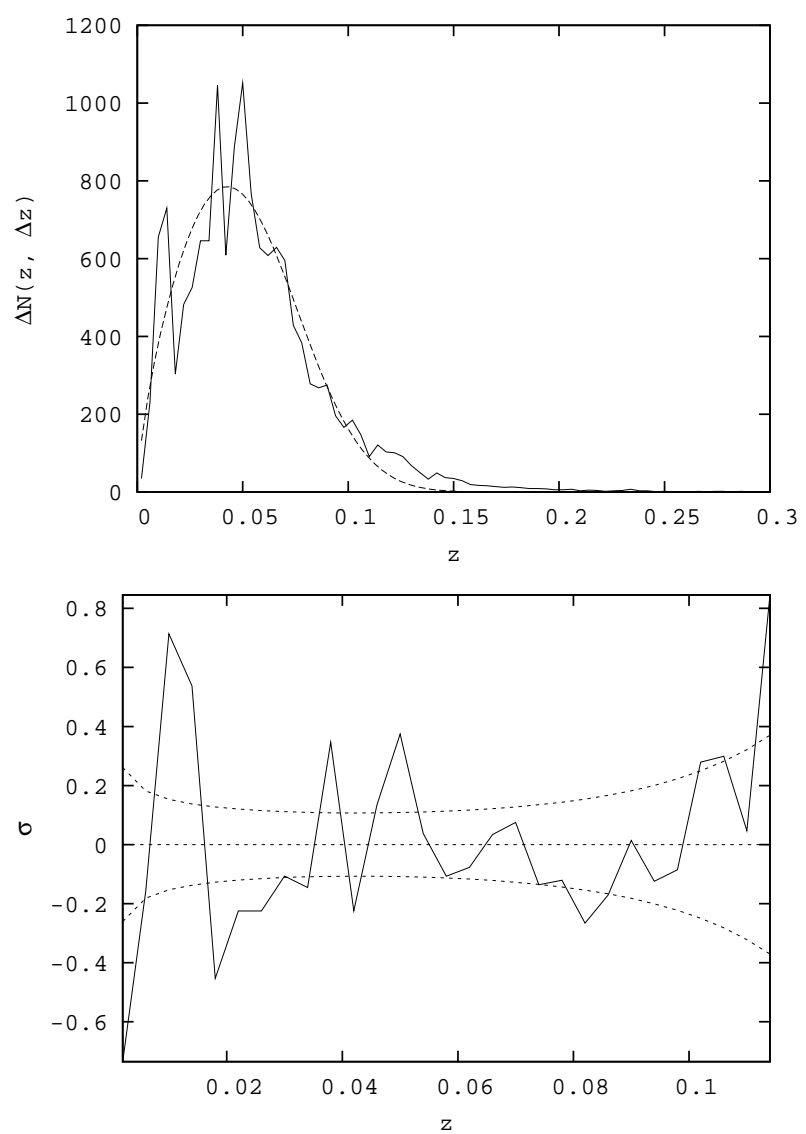

Fig. 9. Radial distribution (top) and the curve of relative fluctuations (bottom) for the 6dF catalog. The first domain $\Delta z=z_{m} / 75$. 
- the empirical formula (2), which is often used to approximate radial distributions of objects in magnitude-limited catalogs, yields equally adequate minimum root-mean-square approximation for both uniform and fractal distributions with dimensions exceeding 2.0. At smaller dimensions the scatter becomes too large and the formula is inapplicable.

We found the fractal dimension to correlate with the deviation of the true radial distribution from the approximating formula, and not with the parameters of the best-fit approximation.

Our analysis of real catalogs yielded the following results:

- the data of the $2 \mathrm{dF}$ catalog imply a fractal dimension of $2.20 \pm 0.25$ in the interval from 2 to $20 \mathrm{Mpc}$. No reliable conclusions can be made on larger scales about the dimension and scale of irregularities due to the intrinsic biases of the method. Deeper surveys and surveys with better sky coverage are needed for this task.

Because of its incompleteness, the $6 \mathrm{dF}$ catalog can not yet be used to derive a reliable estimate for the fractal dimension;

- An analysis of radial distributions revealed the significant irregularities both in the $2 \mathrm{dF}$ and $6 \mathrm{dF}$ catalogs. Deviations from smooth distribution exceed $7 \sigma_{p}$ and their scale lengths amount to $70 \mathrm{Mpc}$. The scale length and magnitude of irregularities correlate rather well with fractal-dimension estimates in the 2.1-2.4 interval.

\section{ACKNOWLEDGMENTS}

I am sincerely grateful to Yu. V. Baryshev for formulating the problem, for assistance and constant attention to this work, and to V. P. Reshetnikov for his useful advices and assistance in preparing the paper. This work was supported by the Russian Foundation for Basic Research (grant no. 09-02-00143).

\section{REFERENCES}

[1] P. J. E. Peebles, astro-ph/0103040

[2] Yu. V. Baryshev, P. Teerikorpi, Bull. Spec. Astrophys. Obs. 59, 92, 2006

[3] N. V.Vasil'ev, MSc thesis (St.-Petersburg State University, 2004).

[4] P. Pirin Erdoğdu, O. Lahav, J. P. Huchra, M. Colless, et al., Monthly Notices Roy. Astronom. Soc. 373, 45 (2006).

[5] R. Massey, J. Rhodes, A. Leauthaud, et al., Astrophys. J. Suppl. 172, 239 (2007).

[6] R. S. Somerville, K. Lee, H. C. Ferguson, et al., Astrophys. J. 600, 171 (2004).

[7] G. S. Busswell, T. Shanks, W. J. Frith, et al., Monthly Notices Roy. Astronom. Soc. 354, 991 (2004).

[8] W. J. Frith, G. S. Busswell, R. Fong, N. Metcalfe and T. Shanks, Monthly Notices Roy. Astronom. Soc. 345, 1049 (2003). 
[9] M. Colless et al., astro-ph/0306581

[10] D. H. Jones, W. Saunders, M. Colless, et al, Monthly Notices Roy. Astronom. Soc. 355, 747 (2004).

[11] D. H. Jones, W. Saunders, M. Read and M. Colless, PASA 22, 277 (2005).

[12] K. Wakamatsu, M. Colless, T. Jarrett, Q. Parker, W. Saunders and F. Watson, ASPC. 289, 97 (2003).

[13] M. Matsumoto and T. Nishimura, ACM Transactions on Modeling and Computer Simulation, 8, 1 (1998).

[14] J. E. Felten, IAUS. 117, 111 (1987).

[15] Y. Yoshii and F. Takahara, Astrophys. J. 326, 1 (1998).

[16] P. Norberg, S. Cole, C. M. Baugh, et al., Monthly Notices Roy. Astronom. Soc. 336, 907 (2002).

[17] F. Sylos Labini, M. Montuori and L. Pietronero, Phys. Rep. 293, 61 (1998).

[18] A. V. Tikhonov et al., Bull. Spec. Astrophys.Obs. 50, 39, 2000. 